\title{
Controlled Crystalline Structure and Surface Stability of Cobalt Nanocrystals
}

\author{
Yuping Bao, Michael Beerman, Alexandre B. Pakhomov, and Kannan M. Krishnan* \\ Materials Science \& Engineering, University of Washington, Seattle, Washington 98195 \\ Received: December 10, 2004; In Final Form: February 10, 2005
}

\begin{abstract}
The synthesis of monodispersed $10 \mathrm{~nm}$ cobalt nanocrystals with controlled crystal morphology and investigation of the surface stability of these nanocrystals are described. Depending on the surfactants used, single crystalline or multiple grain nanocrystals can be reproducibly produced. The relative surface stability of these nanocrystals is analyzed using the temperature dependences of the dc magnetic susceptibility. The novel method, which allows sensitive monitoring of the surface stability, is based on the observation that, with particle oxidation, an anomalous peak appears at $8 \mathrm{~K}$ in zero-field-cooled magnetization measurements. It is found that the surfactant protective layer is more important for long-term stability at room temperature, while the hightemperature oxidation rate is controlled by the crystal morphology of the nanoparticles.
\end{abstract}

\section{Introduction}

A variety of chemical synthesis routes have been investigated to produce metallic nanoparticles with controlled size and shape and uniform size distribution. For example, silver nanocubes, ${ }^{1}$ cobalt nanodiscs, ${ }^{2,3}$ and iron nanorods ${ }^{4}$ have been synthesized. It is believed that a surfactant or polymer plays an important role in the metallic nanoparticles synthesis and, particularly, in their shape control. ${ }^{5}$ However, for metallic nanocrystals, little attention has been given to the effects of the surfactant on the morphology and surface stability of metallic nanocrystals. Furthermore, the long-term stability, especially the propensity of their surfaces to oxidize, is one of the main concerns for applications. Until now, X-ray diffraction (XRD) has been predominantly used to detect oxidation; however, the sensitivity is limited by the thickness of the oxidation layer, the size of the nanocrystals, and the quantity of the samples. Here, we demonstrate our ability to produce cobalt nanoparticles with uniform size distribution and controlled morphologies and surface chemistry. It is also shown that, for metallic cobalt nanocrystals, the surface stability can be sensitively monitored by superconducting quantum interference device (SQUID) measurements, using a small fraction of the sample required for XRD measurement. The relative importance of surface chemistry and crystal morphology for resistance to oxidation is investigated.

\section{Experimental Section}

Synthesis of cobalt nanocrystals was performed under an argon atmosphere and involved the rapid decomposition of cobalt carbonyl in a coordinated solvent containing different surfactants. All the chemicals were obtained from commercial sources and used without further purification. Cobalt nanocrystals, $\sim 10 \mathrm{~nm}$ in diameter, were prepared by following one of these two procedures. (a) TOPO-related reaction (Co@TOPO): $1.58 \mathrm{mmol}$ (0.54 g) of cobalt carbonyl dissolved in $3 \mathrm{~mL}$ of 1,2-dichlorobenzene (DCB) was injected into DCB $\left(182{ }^{\circ} \mathrm{C}\right)$ containing a mixture of $0.6 \mathrm{mmol}(0.2 \mathrm{~mL})$ of oleic acid and $0.26 \mathrm{mmol}(0.1 \mathrm{~g})$ of trioctylphosphine oxide

* Corresponding author. E-mail: kannanmk@u.washington.edu.
(TOPO) and refluxed for $15 \mathrm{~min}$. (b) DOA-related reaction (Co@DOA): $1.58 \mathrm{mmol}(0.54 \mathrm{~g})$ of cobalt carbonyl dissolved in $3 \mathrm{~mL}$ of DCB was injected into DCB $\left(182{ }^{\circ} \mathrm{C}\right)$ containing a mixture of $0.6 \mathrm{mmol}(0.2 \mathrm{~mL})$ of oleic acid and $1.1 \mathrm{mmol}(0.34$ $\mathrm{mL}$ ) of dioctylamine (DOA) and refluxed for $15 \mathrm{~min}$. Both TOPO- and DOA-related recipes yield about $10 \mathrm{~nm}$ cobalt nanocrystals with narrow size distribution. The crystal structure was studied with XRD and transmission electron microscopy (TEM). TEM samples were prepared by evaporating a drop of the particle solution on a TEM grid. Magnetic properties were investigated by SQUID magnetometry. Powder samples for both XRD and SQUID were prepared by precipitating from solution by using methanol, a nonsolvent.

\section{Results and Discussions}

Bright-field TEM images of cobalt nanoparticles grown in a TOPO-related reaction show relative uniform contrast across individual particles, implying a homogeneous crystalline structure of this phase (Figure 1A). High- resolution TEM (HRTEM) images clearly show uniform lattice fringes (Figure 1B) corresponding to (200) lattice planes of the $\epsilon$-Co unit cell. Brightfield TEM and HRTEM images of $10 \mathrm{~nm}$ cobalt nanocrystal grown in a DOA-related reaction are shown in Figure 1C,D. The nanoparticles are comprised of mutiple crystal grains with clear grain boundaries as seen in HRTEM. To verify the bulk structural properties, XRD measurements on powder samples were performed. Both the peak position and the relative peak intensity suggest the cobalt nanocrystals are $\epsilon$-cobalt (Figure 2). ${ }^{6}$ However, the peak broadening for Co@DOA indicates the presence of much smaller crystallites in general agreement with TEM observations. Simulations of XRD spectra suggest that the average gain size for single-crystalline particles is about 12 $\mathrm{nm}$ and $5 \mathrm{~nm}$ for multiple-grained nanoparticles (Supporting Information).

The two different morphologies of the nanocrystals can be explained by considering the functional groups of the different surfactants used in the synthesis. The binding strength of the surfactant to cobalt surface and the characteristic of the surfactant chains affect the monomer activity and concentration of particle nuclei. ${ }^{7}$ The existing monomer concentration after nucleation plays a key role in determining the resulting crystal 


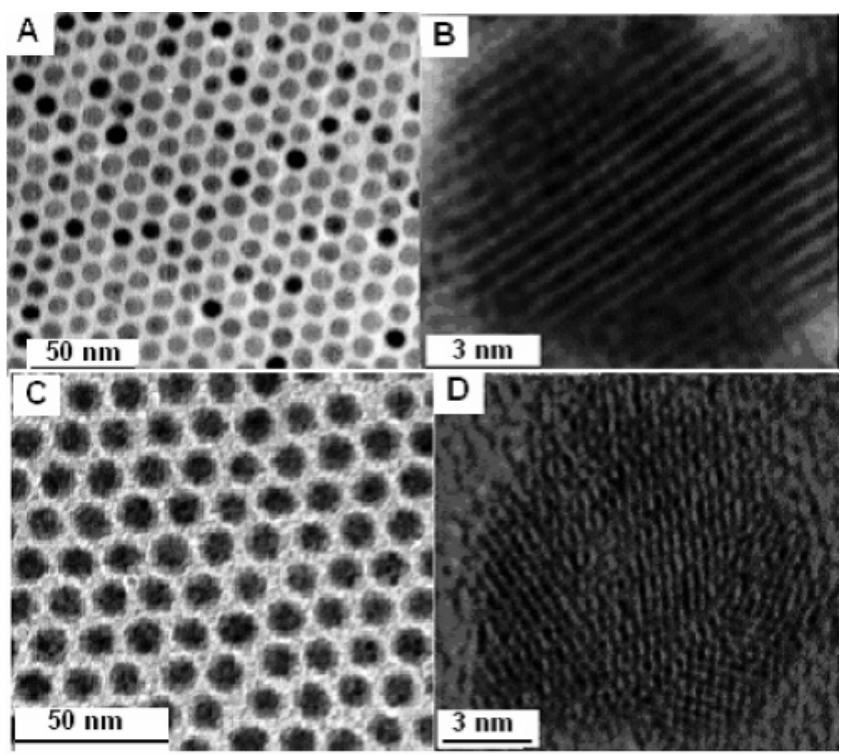

Figure 1. (A) Typical TEM image and (B) lattice image of $10 \mathrm{~nm}$ cobalt nanocrystals grown in a TOPO-related reaction. (C) Typical TEM image and (D) HRTEM image of $10 \mathrm{~nm}$ nanoparticles grown in a DOArelated reaction.

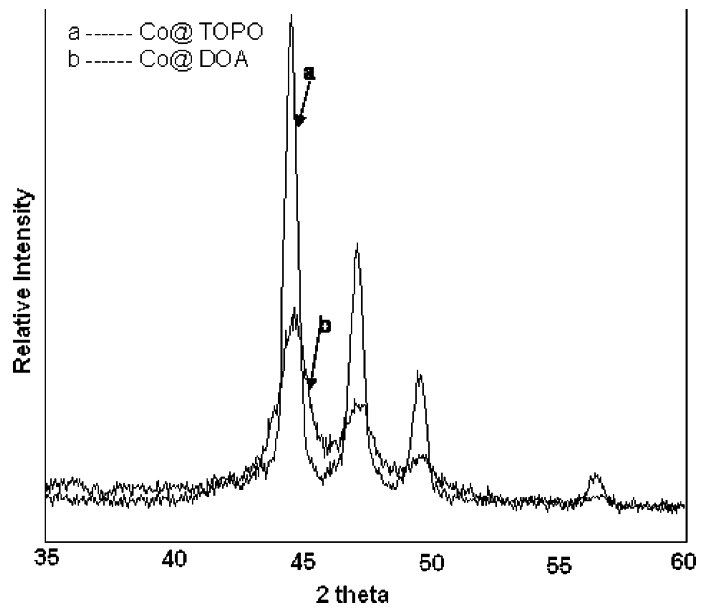

Figure 2. XRD patterns of $10 \mathrm{~nm}$ cobalt nanocrystals, both corresponding to $\epsilon$-Co (space group $P 4_{3} 32$, lattice parameter $6.09 \AA$ ): ${ }^{2}$ (A) nanocrystals grown in a TOPO-related reaction; (B) nanocrystals grown in a DOA-related reaction.

structure $^{8}$ due to the chemical potential of the reaction solution. Our experimental data show that, in addition to TOPO and DOA, oleic acid is required to obtain a specific-size, monodisperse cobalt nanocrystals; here oleic acid is used to prevent the occurrence of Ostwald ripening. TOPO surfactant has a higher bonding strength to the cobalt surface and lower diffusion coefficient because of the bulkier chains when compared to DOA. ${ }^{9}$ For the same amount of stock solution injection, the TOPO-related reaction produces fewer nuclei because of the lower monomer reactivity, resulting in higher excess monomers. Therefore, the chemical potential of the solution is higher, favoring the formation of single crystals. However, in the DOArelated reaction, the monomer reactivity is higher due to the weaker bonding strength of the amine group and less bulky tail, which yields relative high concentration of nuclei. Therefore, the monomer concentration will be quickly depleted and will lead to the formation of small aggregates. Subsequently, these aggregates will join to lower the surface energy resulting in the formation of the multiple-grain structures. This process,
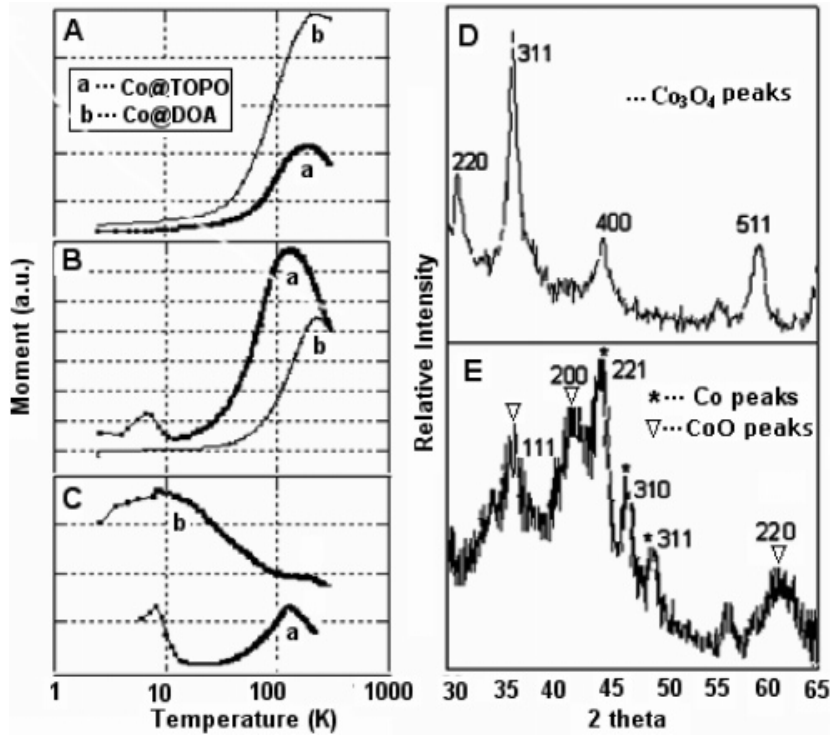

Figure 3. ZFC-FC measurements of two types of cobalt nanocrystals: (A) freshly made samples; (B) 3-month-old samples; (C) after annealing at $140{ }^{\circ} \mathrm{C}$ for $24 \mathrm{~h}$. XRD patterns of nanoparticles after annealing: (D) Co@DOA sample, the peaks after annealing corresponding to $\mathrm{Co}_{3} \mathrm{O}_{4}$; (E) Co@TOPO sample. Except for cobalt oxide peaks, which can be partially indexed as $\mathrm{CoO}, \epsilon-\mathrm{Co}$ peaks are still clearly seen.

known as aggregation of subunits mechanism theory, has been discussed in detail in the literature. ${ }^{10}$

Surface stability differs between these two types of cobalt nanocrystals, depending on the surface chemistry and morphology. The main mechanism of structural decay is due to the formation of a cobalt oxide surface layer. However, XRD does not show sufficient signal from the oxide when the oxide layer is thin. To study the stability of these crystals, zero-field-cooled (ZFC) and field-cooled (FC) measurements of magnetization as a function of temperature in the applied field $H=20 \mathrm{Oe}$ were performed on a Quantum Design SQUID magnetometer. The results for freshly made samples, samples aged for 3 months at room temperature in the air, and 3-month old samples annealed at $140{ }^{\circ} \mathrm{C}$ for $24 \mathrm{~h}$ are shown in Figure 3. The ZFC magnetization of both freshly made samples (Figure $3 \mathrm{~A}$ ) has a maximum near the blocking temperature $T_{B} \sim 220 \mathrm{~K}$ expected for $10 \mathrm{~nm}$ cobalt nanocrystals. ${ }^{11}$ Measurements on samples aged for 3 months at room temperature showed almost no changes in the properties of DOA-coated powders. However, an additional peak in ZFC magnetization at $8 \mathrm{~K}$ appears for the TOPO-coated nanoparticles, and the blocking peak shifts to lower temperature, suggesting the decrease of magnetic size (Figure 3B). After the sample was annealed at $140{ }^{\circ} \mathrm{C}$ for $24 \mathrm{~h}$, this $8 \mathrm{~K}$ peak appears in both samples. Moreover, the blocking peak of the DOA-coated sample has nearly disappeared after annealing, while the TOPO-coated powder has a relatively strong blocking peak along with the low-temperature peak. The blocking peak is shifted to lower temperature due to the decrease of the effective magnetic volume of the particles (Figure 3C). The XRD pattern of DOA-related samples after annealing corresponds to the crystal structure of $\mathrm{Co}_{3} \mathrm{O}_{4}$ (space group $F d 3 m$, lattice parameter $0.808 \mathrm{~nm}$ ); however, for the TOPO-related sample, besides cobalt oxide peaks, which can be partially indexed as $\mathrm{CoO}, \epsilon$-Co peaks are still clearly seen (Figure 3D,E). Thus, the $8 \mathrm{~K}$ peak is a signature of the cobalt oxide layer and can be used as a tool for detecting even the early stages of surface oxidation. 
We have performed a number of magnetic investigations to understand the origin of the $8 \mathrm{~K}$ peak. Measurements on many aged samples of TOPO-coated Co particles of different dimensions in the range 5-20 $\mathrm{nm}$ showed the universal nature of this peak, which always appears at $8 \mathrm{~K}$. The position of this peak does not change with the thickness of the oxide layer. So this peak is not due to blocking phenomenon where the blocking temperature scales with the volume. $\mathrm{Co}_{3} \mathrm{O}_{4}$ is antiferromagnetic with the Neel temperature much higher than $8 \mathrm{~K}$, and reduction of the Neel temperature to $8 \mathrm{~K}$ due to size effect can be ruled out due to the same reason (size independence). To check the possibility of spin-glass-like freezing of frustrated surface moments ${ }^{12}$ at $8 \mathrm{~K}$, we measured the nonlinear susceptibility, which did not show the anomaly at the transition temperature, as should be expected for a spin glass at the freezing temperature. ${ }^{13}$ Finally, fully oxidized samples show ferromagnetictype hysteresis below this temperature. On the other hand, the $8 \mathrm{~K}$ peak is readily observed in $\mathrm{ZFC}$ measurements of commercial micrometer-sized $\mathrm{Co}_{3} \mathrm{O}_{4}$ powder. We conclude that we observe either ferromagnetic ordering in the surface layer of uncompensated spins or a metamagnetic transition from antiferromagnetic to ferromagnetic state in $\mathrm{Co}_{3} \mathrm{O}_{4}$. Anomalous behavior of magnetic properties at low temperature has been observed in $\mathrm{Ni}$ and $\mathrm{Co}$ ferrite nanoparticles ${ }^{14,15}$ and has been discussed in terms of a model of particles consisting of ferrimagnetically aligned core spins and a spin-glass-like surface layer, ${ }^{14}$ an extra contribution of surface spin moments to the total magnetization has been considered. ${ }^{15}$ Nevertheless, in our case, independent of its origin, the appearance of the $8 \mathrm{~K}$ peak clearly indicates the onset of the oxidation of Co.

The results in Figure 3 show that, at high temperatures, the TOPO-coated particles are more stable than DOA-coated particles. But at room temperature DOA protects the nanoparticles better. These results can be explained by considering the properties of surfactant chains and metal cores in these two different cases. The role of surfactant in protecting Co nanoparticles from oxidation may be explained by considering the steric hindrance of the surfactant. The higher steric hindrance of TOPO surfactant makes it difficult for surfactant molecules to form a stable close packing on the surface, and the surfactant layer is penetrable by oxygen. In contrast, the higher flexibility of DOA allows the molecules to rearrange themselves and fully bind to the cobalt surface and form a good protective layer. At room temperature, packing density seems to be important; hence, TOPO-coated particles appear more oxidized after 3 months of exposure to air, as indicated by the low-temperature magnetization peak, than the DOA-coated particles (Figure 3B). After the samples were annealed at temperature high enough to accelerate the oxidation of cobalt but low enough to keep the surfactants stable, oxidation occurs in both samples (Figure 3C). The TOPO-related sample still has a relatively strong blocking peak, which means that the metal cores have mostly survived. However, the DOA-related sample has oxidized almost completely and blocking is barely visible. In this case neither of the coatings can protect the surface. Oxidation rate is now determined by diffusion of oxygen into the particle body, which is higher in the case of multiple-grained nanocrystal samples produced in the DOA-related reaction (compare the TEM images in Figure 2).

\section{Conclusions}

In conclusion, we produced single-crystal and multiple-grain $\epsilon$-cobalt nanocrystal structures using two surfactant types in the synthesis. It was shown that ZFC measurements provide a sensitive tool to study the stability of magnetic nanocrystals and especially the onset of surface oxidation. Using this method we demonstrated that at room temperature the surfactant protective layer and, alternatively, at higher temperature the crystal morphology (single-crystal or multiple-grained nanoparticles) of nanoparticles play the dominant roles in their stability with respect to oxidation.

Acknowledgment. This work is funded by the NSF (Grant DMR-0203069) and the Campbell Endowment at UW. We appreciate the use of TEM facilities at LBNL, PNNL, and UW Zoology.

Supporting Information Available: Group electronegativity of surfactant calculation based on Lewis acid-base theory and Jade software simulation results for two types of cobalt nanoparticles. This material is available free of charge via the Internet at http://pubs.acs.org.

\section{References and Notes}

(1) Sun, S.; Xia, Y. Science 2002, 298, 2176-2179.

(2) Puntes, V. F.; Krishnan, K. M.; Alivisatos, A. P. Science 2001, 291, 2115-2117.

(3) Puntes, V. F.; Zanchet, D.; Erdonmez, C. K.; Alivisatos, A. P. J. Am. Chem. Soc. 2002, 124, 12874-12880. 8582 .

(4) Park, S.; Kim, S.; Lee, S. J. Am. Chem. Soc. 2000, 122, 8581-

(5) Murphy, C. J. Science 2002, 298, 2139-2141.

(6) Murray, C. B.; Sun, S. J. Appl. Phys. 1999, 85, 4325-4330.

(7) Yu, W. W.; Wang, Y. A.; Peng, X. Chem. Mater. 2003, 15, 43004308.

(8) Peng, X. Adv. Mater. 2003, 15, 459-463

(9) TOPO has a higher bonding ability on cobalt surface than DOA surfactant because the functional group $(\mathrm{P}=\mathrm{O})$ has a higher electronegativity $(2.83 \mathrm{eV})$ and two lone electron pairs, whereas the functional group of DOA (N) has lower electronegativity $(2.80 \mathrm{eV})$ and only one electron pair (Supporting Information).

(10) Park, J.; Privman, V.; Matijević E. J. Phys. Chem. B 2001, 105, 11630-11635.

(11) The blocking temperature is determined by the particle volume $V$ and magnetic anisotropy constant $K_{A} \sim 0.21 \mathrm{~J} / \mathrm{m}^{3}$ for $\epsilon$-Co. The relation between these parameters follows from the Neel expression [Neel, L. Ann. Geophys. 1949, 5, 99] $\tau_{\mathrm{m}} \sim \tau_{0} \exp \left[K_{\mathrm{A}} V / k T_{\mathrm{B}}\right]$, where $\tau_{0} \sim 10^{-10} \mathrm{~s}$ is the microscopic fluctuation attempt time and $\tau_{\mathrm{m}} \sim 10 \mathrm{~s}$ is the SQUID measurement time. In the superparamagnetic state above the blocking temperature, the particle moment can rotate during the time of measurement.

(12) Kodama, R. H.; Berkowitz, A. E.; McNiff, E. J.; Foner, S. Phys. Rev. Lett. 1996, 77, 394-397.

(13) Novak, M. A.; Symko, O. G.; Zheng, D. J. Phys. Rev. B 1986, 33, 343-346.

(14) Komada, R. H.; Berkowitz, A. E.; Mcniff, E., J., Jr.; Foner, S. J. Appl. Phys. 1997, 81, 5552-5554.

(15) Tung, L. D.; Kolesnichenko, V.; Caruntu, D.; Chou, N. H.; O’Connor, C. J.; Spinu, L. J. Appl. Phys. 2003, 93, 7486-7488. 